Miami, 1994. MMWR 1994;43:773-775.

50. Centers for Disease Control and Prevention. Human rabiesWest Virginia, 1994. MMWR 1995;44:86-87, 93.

51. Centers for Disease Control and Prevention. Human rabiesAlabama, Tennessee, and Texas, 1994. MMWR 1995;44:269272.

52. Centers for Disease Control and Prevention. Human rabiesWashington, 1995. MMWR 1995;44:625-627.

53. Centers for Disease Control and Prevention. Human rabiesConnecticut, 1995. MMWR 1996;45:207-209.

54. Centers for Disease Control and Prevention. Human rabiesCalifornia, 1995. MMWR 1996;45:353-356.

55. Moellering RC Jr. The Garrod lecture: the Enterococcus: a classic example of the impact of antimicrobial resistance on therapeutic options. J Antimicrob Chemother 1991;28:1-12.

56. Frieden TR, Munsiff SS, Low DE, et al. Emergence of vancomycin-resistant enterococci in New York City. Lancet 1993;342:76-79.

57. Cohen ML. Epidemiology of drug resistance: implications for a post-antimicrobial era. Science 1992;257:1050-1055.
58. Hospital Infection Control Practices Advisory Committee. Recommendations for preventing the spread of vancomycin resistance. $M M W R$ 1995;44(RR-12):1-13.

59. Jernigan DB, Hofmann J, Cetron MS, et al. Outbreak of Legionnaires' disease among cruise ship passengers exposed to a contaminated whirlpool spa. Lancet 1996;347:494-499.

60. Duchin JS, Koster F, Peters CJ, et al. Hantavirus pulmonary syndrome: clinical description of seventeen patients with a newly recognized disease. $N$ Engl J Med 1994;330:949-955.

61. Bell BP, Goldoft M, Griffin PM, et al. A multistate outbreak of Escherichia coli O157:H7-associated bloody diarrhea and hemolytic uremic syndrome from hamburgers: the Washington experience. JAMA 1994;272:1349-1353.

62. Boyce TG, Pemberton AG, Wells JG, Griffin PM. Screening for Escherichia coli 0157:H7-a nationwide survey of clinical laboratories. J Clin Microbiol 1995;33:3275-3277.

63. Tenover FC, Tokars J, Swenson J, Paul S, Spitalny K, Jarvis W. Ability of clinical laboratories to detect antimicrobial agentresistant enterococci. J Clin Microbiol 1993;31:1695-1699.

\title{
Outbreaks of Cyclospora Infections in US and Canada
}

\section{Gina Pugliese, RN, MS; Martin S. Favero, $\mathbf{P h D}$ Medical News Editors}

The CDC recently reported a number of outbreaks of Cyclospora cayetanensis infection in May and June 1996 in at least 10 states in the United States and Ontario, Canada. Cyclospora cayetanensis is a parasite that causes a diarrheal illness. Only three outbreaks have been reported in the United States since it was first diagnosed in humans in 1977.

Several hundred laboratoryconfirmed cases have been reported to the CDC, mostly involving immunocompetent adults. Preliminary evidence suggests that consumption of raspberries and mixtures of berries and other fresh fruits may be associated with Cyclospora infection. The CDC, the FDA, and health officials in the
United States and Canada are collaborating to determine the extent and cause of the outbreaks, the sources of contamination, and whether transmission is ongoing. Additional efforts include testing samples of produce for Cyclospora.

Although Cyclospora is transmitted by the fecal-oral route, direct person-to-person transmission is unlikely, because excreted oocysts require days to weeks under favorable conditions to become infectious (ie, sporulate). The incubation period is 1 week, and illness may be protracted (from days to weeks), with frequent, watery stools and other gastrointestinal symptoms; symptoms may remit and relapse. Oocysts can be identified in the stool by examination of wet mounts under phase microscopy, use of modified acid-fast stains, or demonstration of autofluorescence with ultra- violet epifluorescence microscopy. However, these procedures are not routine for most clinical laboratories and therefore confirmation by an experienced reference laboratory is recommended. Infection can be treated with trimethoprim-sulfamethoxazole.

The specific sources or modes of transmission have not been identified, and the complex distribution routes and handling of seasonal produce complicates tracing. As always, raw produce should be washed thoroughly before eating. Cases should be reported to local and state health departments.

FROM: Centers for Disease Control and Prevention. Outbreaks of Cyclospora cayetanensis infectionUnited States, 1996. MMWR 1996;45(25):549-551. 